\title{
710 DIFFERENCES IN THE SUSCEPTIBILITY OF HUMAN SMALL CELL LUNG CANCER VARIANTS TO NK CELL- MEDIATED LYSIS CAN BE OVERCOME WITH THE ADDITION OF N803 (IL-15 SUPERAGONIST)
}

${ }^{1}$ Kristen Fousek*, ${ }^{1}$ Lucas Horn, ${ }^{1}$ Haiyan Qin, ${ }^{2}$ Bobby Reddy, ${ }^{2}$ Lennie Sender, ${ }^{2}$ Patrick Soon Shiong, 'Jeffrey Schlom, ${ }^{1}$ Claudia Palena. 'National Institutes of Health, Bethesda, MD, United States; ${ }^{2}$ ImmunityBio, Inc., Culver City, CA, United States

Background Small cell lung cancer (SCLC) is a highly aggressive tumor with a 5 -year survival rate of less than 5\%. Traditionally characterized as a neuroendocrine (NE) cancer, several subtypes have now been identified which vary in their phenotypic and transcriptional profiles. Classical NE tumors are molecularly defined as ASCL1+ or NEUROD1+ and exhibit an epithelial phenotype, expressing cytokeratin and E-cadherin (E-Cad). In contrast, non-classical variants express POU2F3 or YAP1 and are enriched in mesenchymal features, such as high levels of vimentin (Vim). Prior studies describe that non-NE variants of SCLC are less susceptible to chemotherapy and may arise via therapeutic selection. With the addition of immune checkpoint blockade to first-line chemotherapy for the treatment of advanced SCLC, understanding whether SCLC variants respond differently to immunotherapy is crucial.

Methods We utilized a range of pre-clinical models to investigate whether molecular and phenotypic variants of SCLC differ in their susceptibility to immune-mediated lysis. Following extensive characterization at the RNA and protein levels for expression of ASCL1, NEUROD1, POU2F3, YAP1, epithelial E-Cad, mesenchymal Vim, and other markers of cell phenotype, a panel of cells including each variant subtype were selected for further study.

Results Upon exposure to healthy donor effector NK cells, the more epithelial cells were highly susceptible to NK-mediated cytotoxicity while all mesenchymal SCLC cells remained highly refractory to NK-mediated lysis. This prompted us to investigate immunotherapy approaches such as the addition of N803, a mutant IL-15 superagonist, to improve the activation and proliferation of NK cells. In a xenograft model utilizing the mesenchymal YAP1+ H841 cell line subcutaneously implanted into nude mice devoid of all immune cells except for NK cells, we observed that the weekly administration of N803 resulted in a significant increase in the number of activated NK cells within the spleens of treated mice. Additionally, NK cells from treated mice produced significantly higher levels of IFN-gamma and granzyme B, resulting in a significant decrease in overall tumor burden.

Conclusions Our data indicates that N803-activated NK cells effectively mediate lysis of SCLC across all variant types, including those previously completely refractory to traditional NK cell lysis. These results highlight the potential of N803 as a novel immune-based intervention for the treatment of all variants of SCLC.

Ethics Approval PBMCs were obtained from healthy donors at the NIH Clinical Center Blood Bank (NCT00001846). All animal studies were approved and conducted in accordance with an IACUC-approved animal protocol (LTIB-57) with the approval the NIH/NCI Institutional Animal Care and Use Committee.

http://dx.doi.org/10.1136/jitc-2021-SITC2021.710 